\title{
Structural analysis by interval approach
}

\author{
Franck Massa - Karine Mourier-Ruffin - Bertrand Lallemand \\ Thierry Tison
}

Laboratoire d'Automatique, de Mécanique et d'Informatique Industrielles et Humaines, UMR 8530, Université de Valenciennes

Le Mont Houy, F-59313 Valenciennes cedex 9

franck.massa@univ-valenciennes.fr

\begin{abstract}
Finite element simulations are well established in industry and are an essential part of the design phase for mechanical structures. Although numerical models have become more and more complex and realistic, the results can still be relatively far from observed reality. Nowadays, use of deterministic analysis is limited due to the existence of several kinds of imperfections in the different steps of the structural design process. This paper presents a general non-probabilistic methodology that uses interval sets to propagate the imperfections. This methodology incorporates sensitivity analysis and reanalysis techniques. Numerical interval results for a test case were compared to experimental interval results to demonstrate the capabilities of the proposed methodology.

RÉSUMÉ. Les simulations par éléments finis sont bien implantées dans le monde industriel et sont devenues incontournables en phase de conception des structures mécaniques. Bien que les modèles numériques soient de plus en plus complexes et réalistes, les résultats obtenus présentent encore des écarts non négligeables par rapport à la réalité observée. L'utilisation d'une analyse déterministe est limitée car elle néglige les imperfections de la phase de fabrication ou encore de la méconnaissance des données d'avant-projet. Une méthodologie générale de nature non probabiliste, utilisant les intervalles, est présentée pour propager les imperfections. Celle-ci est basée sur le couplage entre analyse de sensibilité et technique de réanalyse par projection. Un cas test est présenté pour démontrer la capacité de la méthodologie à prédire un ensemble de résultats expérimentaux.

KEYWORDS: imperfections, interval, reanalysis, frequency response functions, aggregation of experimental results.

MOTS-CLÉS: imperfections, intervalles, réanalyse, fonctions de réponse en fréquence, agrégation de résultats expérimentaux.
\end{abstract}

DOI:10.3166/REMN.17.869-880 @ 2008 Lavoisier, Paris

REMN - 17/2008. Giens 2007, pages 869 to 880 


\section{Introduction}

Many adjectives are frequently associated to the data and/or objectives used in structural analysis: uncertain, imprecise, vague, unknown and subjective are just some examples. The difficulty in characterizing parameters necessarily leads to errors being introduced into deterministic numerical models. In mechanical engineering, such imperfections may be due, for example, to modelling errors, material properties or the geometrical tolerances in manufacturing and assembly. Given this context, the problem of how data imperfection can be taken into account during the design phase has caught the attention of the scientific community.

To quantify behavioral evolutions, many nondeterministic approaches have already been used, namely probabilistic, stochastic, finite element (Sudret et al., 2000) non-parametric (Capiez-Lernout et al., 2006), interval (McWilliam, 2000) and fuzzy set (Massa et al., 2003) methods. The main objective of all these approaches is to precisely quantify the propagation of imperfections in industrial models, while keeping computational costs compatible with the design phase.

This paper proposes a non-probabilistic general approach using interval formalism to propagate imperfections in finite element models. Our approach's objective is to identify the boundaries of a structure's behavioural variation for several kinds of analysis and provide these boundaries to the designer in order to optimize the product and make it more robust (Massa et al., 2006). The reanalysis phase, in which perturbed eigensolutions are approximated, and the propagation phase, in which imperfections are propagated on Frequency Response Functions (FRFs), are explained in detail. Then, numerical and experimental interval FRFs are compared to highlight the capabilities of this approach.

\section{Imperfection quantification}

Interval formalism is employed here to manage imperfection in structural analysis applications. Each parameter of a numerical model, defined with imperfections, is described by an interval and is indexed by the symbol ${ }^{I}$. The interval parameter $P^{I}$ is usually written in the following form:

$$
P^{I}=[\underline{P} ; \bar{P}]=\left[P^{c}+\underline{\Delta P} ; P^{c}+\overline{\Delta P}\right]
$$

where $\underline{P}, \bar{P}, P^{c}, \underline{\Delta P}, \overline{\Delta P}$ are respectively the lower bound of the interval, the upper bound of the interval, the nominal value, the lower variation and the upper variation.

The interval concept and arithmetic first appeared in the mid-1960s (Moore, 1966; Alefeld et al., 1983). Although interval mathematical algorithms have been proposed for the last forty years to solve linear interval equations, nonlinear interval equations and interval eigenvalue problems, it is difficult to apply these results 
directly to practical engineering problems in which large finite element matrices must be managed. In addition, with classic interval arithmetic, the interval solution is always overestimated because of multi-occurrence problems that prevent the boundaries of a structure's behavioral variation from being predicted precisely.

When interval parameters are considered, the parameter variation space is a hyper volume whose size is a function of the number of interval parameters. From a practical viewpoint, this continuous variation space can be discretized by subdividing the interval associated to each interval parameter. The interval parameter $P^{I}$ is defined as a set of discrete values:

$$
P^{I}=\left\{P^{1}, P^{2}, \ldots, P^{n d}\right\}
$$

where $n d$ is the number of subdivisions. To build interval solutions, the problem becomes finding, among all the different combinations of parameter values, those that lead to the minimum and maximum solution variations. A variation space and the associated response surface are illustrated Figure 1 for the case of two interval parameters.

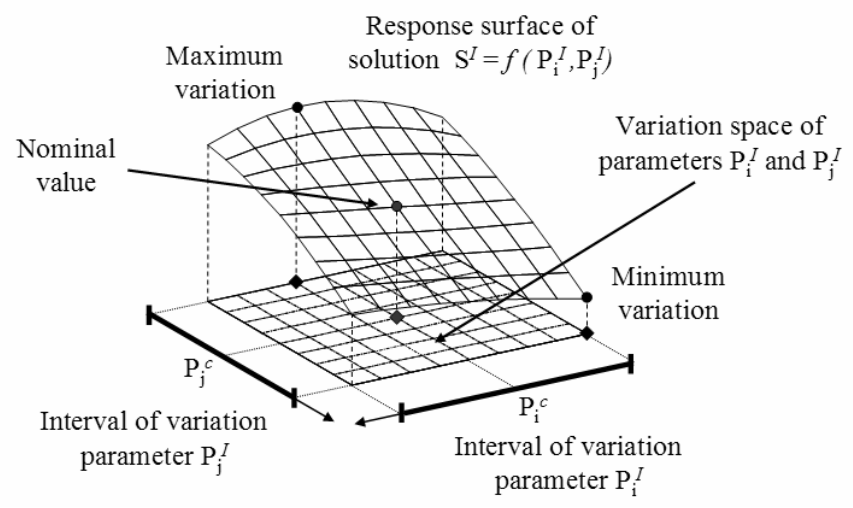

Figure 1. Space of variation for 2 interval parameters

The computation time needed to calculate all the possible parameter value combinations can be considerable. This time can be reduced by studying the solutions' functional dependence with respect to the parameters and by implementing an appropriate methodology. Indeed, depending on the type of solutions studied (e.g., displacements, stresses, eigenvalues, eigenvectors) and the fuzzy parameters (e.g., Young's modulus, Poisson's ratio, density), the nature of functional dependence is not always the same. This dependence may or may not be monotonic (e.g., linear, quadratic). For certain parameters, simple rules can be defined because the solutions' functional dependence is monotonic. For example, eigenvalues tend to increase as Young's modulus increases and decrease as density increases. However, 
for other parameters, such as Poisson's ratio or plate thickness, the functional dependence is not always monotonic, particularly in the case of large variations. These parameters are present in both the mass and stiffness matrices, either defined by a rational fraction in the case of Poisson's ratio, or as a function of power in the case of plate thickness. For these kinds of parameters, the functional dependence is different for each eigenvalue and for each component of the solution vector.

Practically, CPU time can be reduced in different ways, either by limiting the number of combination calculations, or by substituting an efficient approximation for the finite element calculations of perturbed solutions. In the best case scenario, both can be done at the same time. The authors (Massa, 2005; Massa et al., 2003) have already proposed an Interval or Fuzzy Finite Element Method (IFEM or FFEM) to deal with the interval or fuzzy static and modal analysis. This method is reviewed briefly in the next section. In section 5, we propose extending the IFEM to the case of interval dynamic problem by basing our investigation on our previous research about modal interval analysis.

\section{Imperfection propagation}

As mentioned above, the authors (Massa, 2005; Massa et al., 2003) have already proposed a method for solving static and modal interval finite element problems. This method has two steps: 1) the determination of the parameter value combinations that lead to extreme solution variations and 2) the approximation of extreme solutions for these specific combinations in order to build the interval solution.

The interval problem $S^{I}=f\left(P_{1}^{I}, \ldots, P_{N p}^{I}\right)$ is transformed into two optimization problems in which the minimum and maximum solution variations are sought:

$$
\begin{aligned}
S^{I}=[\min \{ & \left.f\left(P_{1}^{1}, \ldots, P_{N p}^{1}\right), \ldots, f\left(P_{1}^{1}, \ldots, P_{N p}^{n d}\right), \ldots, f\left(P_{1}^{n d}, \ldots, P_{N p}^{n d}\right)\right\} \\
& \left.\max \left\{f\left(P_{1}^{1}, \ldots, P_{N p}^{1}\right), \ldots, f\left(P_{1}^{1}, \ldots, P_{N p}^{n d}\right), \ldots, f\left(P_{1}^{n d}, \ldots, P_{N p}^{n d}\right)\right\}\right]
\end{aligned}
$$

where $N_{p}$ is the number of interval parameters. As the functional dependence is not highly nonlinear, these combinations of parameter values associated to extreme variations can be identified by performing sensitivity analyses at different points in the parameter space. (More details of this strategy can be found in Massa et al., 2005). For each identified combination, a high order approximation-for example Taylor expansion (Massa et al., 2006) and more recently Padé approximants (Massa et al., 2008) — is used to limit the computational cost.

In order to improve the precision of the approximation, particularly in the case of eigenvectors, we propose a reanalysis technique using projection. This technique seeks approximate solutions in a subspace $T$, which is independent of parameters $P_{i}$, 
by solving for each value of the parameters $P_{i}$. In the case of modal analysis, the equilibrium equation becomes:

$$
\left[T^{T} K(P) T\right] q=\left[T^{T} M(P) T\right] q \Lambda
$$

where $\Psi$ and $q$ are, respectively, the modal basis and the modal coordinates. Equation [4] is solved very quickly, and then the responses for all degrees of freedom are easily restituted using $\Psi=T q$. As underlined by (Balmès et al., 2005), the fundamental issue is determining the procedure for building a subspace $T$ that will provide good predictions for all desired values of $P_{i}$. We suggest constituting this subspace $T$ with the nominal solutions for the studied problem and the orthonormalized perturbed solutions used to construct the rational fractions for Padé approximants (Cochelin et al., 1994). The subspace $T$ is written:

$$
T=\left[\phi_{1}^{0}, \ldots, \phi_{1}^{N}, \ldots, \phi_{i}^{0}, \ldots, \phi_{i}^{N}, \ldots\right]
$$

where $\phi_{j}^{k}$ is the $j^{\text {ème }}$ perturbed eigenvector of order $\mathrm{k}$. The perturbed eigenvectors are obtained by introducing a perturbation into the mass and stiffness matrices and using Lee's method (Lee et al., 1997):

$$
\begin{aligned}
& \left(\mathbf{K}_{0}+\varepsilon \boldsymbol{\Delta} \mathbf{K}\right)\left(\phi_{0}+\varepsilon \phi_{1}+\ldots+\varepsilon^{\mathrm{n}} \phi_{n}\right)=\ldots \\
& \left(\lambda_{0}+\varepsilon \lambda_{1}+\ldots+\varepsilon^{\mathrm{n}} \lambda_{n}\right)\left(\mathbf{M}_{0}+\varepsilon \boldsymbol{\Delta} \mathbf{M}\right)\left(\phi_{0}+\varepsilon \phi_{1}+\ldots+\varepsilon^{\mathrm{n}} \phi_{n}\right) \\
& \mathbf{K}_{0} \phi_{0}=\lambda_{0} \mathbf{M}_{0} \phi_{0} \\
& {\left[\begin{array}{cc}
\mathbf{K}_{0}-\lambda_{0} \mathbf{M}_{0} & -\mathbf{M}_{0} \phi_{0} \\
-\phi_{0}^{T} \mathbf{M}_{0} & 0
\end{array}\right]\left\{\begin{array}{l}
\phi_{1} \\
\lambda_{I}
\end{array}\right\}=\left[\begin{array}{c}
-\left(\Delta \mathbf{K}-\lambda_{0} \mathbf{\Delta} \mathbf{M}\right) \phi_{0} \\
0.5 \phi_{0}^{T} \mathbf{\Delta} \mathbf{M} \phi_{0}
\end{array}\right]} \\
& {\left[\begin{array}{cc}
\mathbf{K}_{0}-\lambda_{0} \mathbf{M}_{0} & -\mathbf{M}_{0} \phi_{0} \\
-\phi_{0}^{T} \mathbf{M}_{0} & 0
\end{array}\right]\left\{\begin{array}{c}
\phi_{2} \\
\lambda_{2}
\end{array}\right\}=\left[\begin{array}{c}
-\left(\Delta \mathbf{K} \phi_{1}-\lambda_{0} \mathbf{\Delta} \mathbf{M} \phi_{1}-\lambda_{1} \mathbf{\Delta} \mathbf{M} \phi_{0}-\lambda_{1} \mathbf{M}_{0} \phi_{1}\right) \\
0.5\left(\phi_{0}^{T} \mathbf{\Delta} \mathbf{M} \phi_{1}+\phi_{1}^{T} \mathbf{\Delta} \mathbf{M} \phi_{0}+\phi_{1}^{T} \mathbf{M}_{0} \phi_{1}\right)
\end{array}\right]}
\end{aligned}
$$

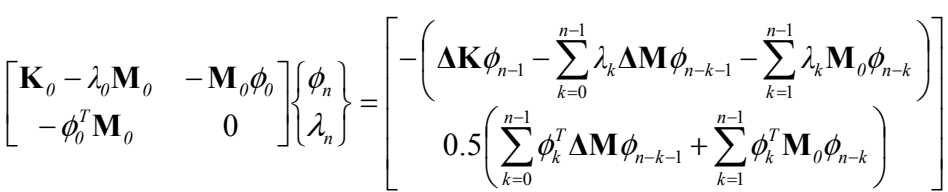

where $\varepsilon$ represents an additional unknown that allows the perturbed problem to be defined. This unknown is similar to the control parameter of classic iterative algorithms.

This reanalysis technique is very powerful even when the variations of mode shape behavior are significant. To illustrate this remark, Figure 2 shows an imperfection of $30 \%$ introduced on thicknesses $\mathrm{e}_{1}$ and $\mathrm{e}_{5}$ in the structure represented. This structure is very sensitive to the variations of different plate thicknesses. The 
Modal Assurance Criterion (MAC), calculated between the nominal and perturbed modal bases, shows the importance of this modification. For example, the variation of the mode 5 components is close to $70 \%$ with a MAC of 0.5 . Comparing the reference eigensolutions (finite element calculations) and approximated eigensolutions confirms the efficiency of this reanalysis technique: the maximum error in the eigensolutions is inferior to $0.05 \%$.

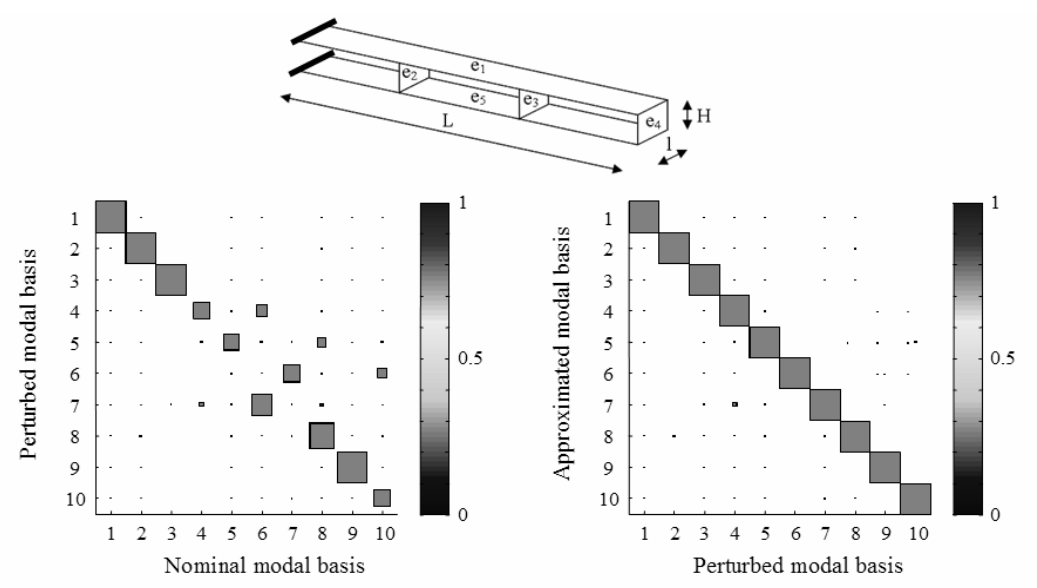

Figure 2. Cantilever box beam and results of approximation

\section{Interval modal superposition}

Consider now a system defined with two interval parameters $P_{1}$ and $P_{2}$. The FRFs obtained for certain combinations of discrete values of interval parameters are shown in Figure 3. Three specific kinds of zones are distinguished in the figure: resonance zones (2, 6 and 9), antiresonance zones (5) and intermediary zones (1, 3, 4, 7, 8 and 10).

Examining the response surfaces pictured in Figure 4 highlights two main cases of functional dependence for these three specific kinds of zones:

On the one hand, the functional dependence is quite non-linear for the resonance (response surfaces B \& E) and antiresonance (response surface D) zones. The extreme variations are not obtained for the combinations of extreme values of interval parameters. Studying the response surfaces for successive frequencies shows that extreme behavioral variations are obtained for different combinations of discrete values of interval parameters. In this case, determining the extreme variation for all these zones requires that almost all the combinations of interval parameter values be calculated. On the other hand, the functional dependence is generally linear or quadratic in the intermediary zones (response surfaces A, C and F) as is the case for modal analysis. 


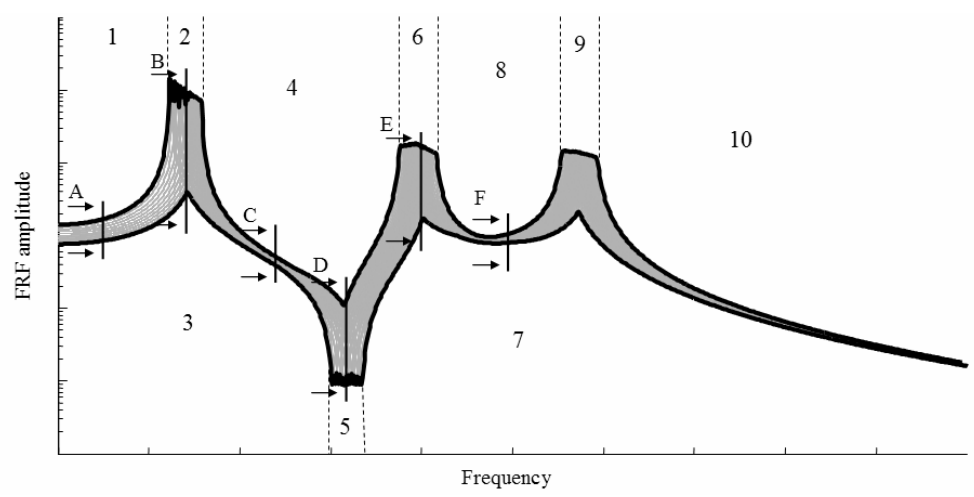

Figure 3. Example of interval FRF
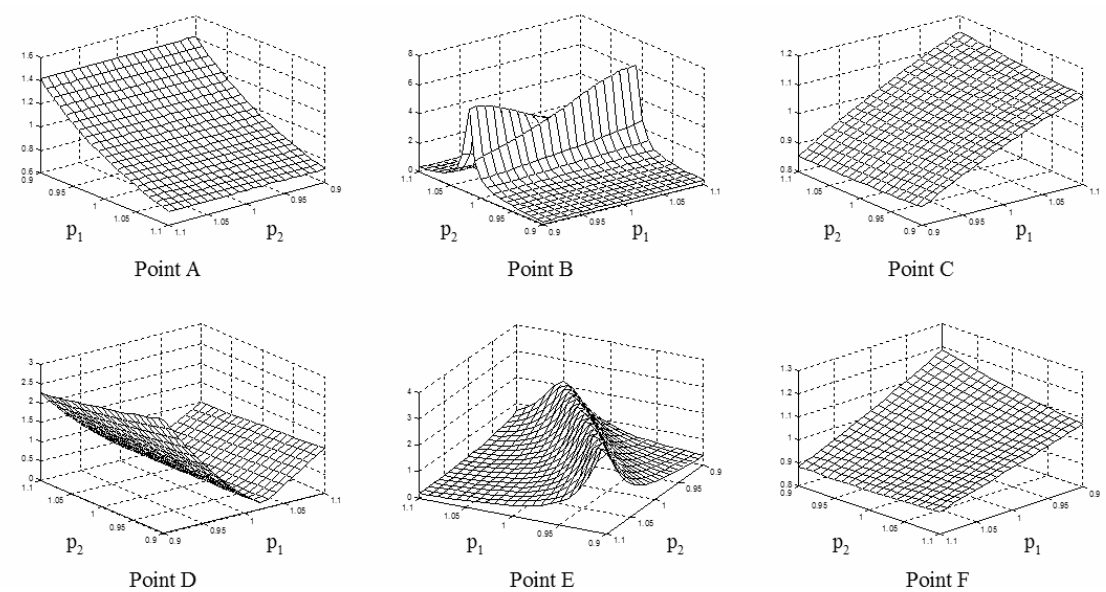

Figure 4. Response surfaces for different frequency steps

This simple parametric analysis is representative of the functional dependence of solutions and the modification of the dynamic behavior of general mechanical structures described with imperfection. Using IFEM (Section 3) for each frequency step will be inevitably time consuming. In order to reduce the CPU time, we studied the modal superposition formula, in the case of modal damping, to determine the number of modal parameter value combinations that will be useful to define the variation boundaries of the FRFs. The FRF observed at point $i$ for $m$ modes and $k$ excitations is written: 


$$
X_{i}^{I}(\Omega)=\sum_{v=1}^{m} \frac{\Phi_{i v}^{I} \cdot \sum_{j=1}^{k} \Phi_{j v}^{I} \cdot F_{j}^{I}}{\omega_{0 v}^{I}+2 . j \cdot \xi_{v}^{I} \cdot \omega_{0 v}^{I} \cdot \Omega-\Omega^{2}}
$$

where $\omega_{0 v}{ }^{I}, \xi_{v}{ }^{I}$ and $\Phi_{v}{ }^{I}$ represent respectively the $v^{\text {ème }}$ eigenpulsation, the $v^{\text {ème }}$ damping ratio, the $v^{\text {eme }}$ eigenvector, the loading vector $F^{I}$, and $\Omega$ the excitation pulsation.

To detect specific parameter combinations, the square of the module of Equation [8] is analyzed for each zone, namely the resonance, antiresonance and intermediary zones:

$$
\begin{gathered}
\left\|X_{i}^{I}(\Omega)\right\|^{2}=\left[\sum_{v=1}^{m} \frac{\left(\Phi_{i v}^{I} \cdot \sum_{j=1}^{k} \Phi_{j v}^{I} \cdot F_{j}^{I}\right) \cdot\left(\omega_{0 v}^{I}{ }^{2}-\Omega^{2}\right)}{\left(\omega_{0 v}^{I}{ }^{2}-\Omega^{2}\right)^{2}+\left(2 \cdot \xi_{v}^{I} \cdot \omega_{0 v}^{I} \cdot \Omega\right)^{2}}\right]^{2}+ \\
{\left[\sum_{v=1}^{m} \frac{\left(\Phi_{i v}^{I} \cdot \sum_{j=1}^{k} \Phi_{j v}^{I} \cdot F_{j}^{I}\right) \cdot\left(2 \cdot \xi_{v}^{I} \cdot \omega_{0 v}^{I} \cdot \Omega\right)}{\left(\omega_{0 v}^{I}{ }^{2}-\Omega^{2}\right)^{2}+\left(2 \cdot \xi_{v}^{I} \cdot \omega_{0 v}^{I} \cdot \Omega\right)^{2}}\right]^{2}}
\end{gathered}
$$

A sensitivity analysis of the module of Equation [8] according to damping ratio showed that this parameter did not have much influence on intermediary zones, which makes it possible to attribute a nominal value to this damping ratio. On the contrary, for the resonance zone, a specific value must be determined for the damping ratio.

By moving away from the mode's resonance zone (zones 3 and 4), the term $2 . \xi_{v}^{I} . \omega_{0 v}^{I} . \Omega$ becomes negligible according to $\omega_{0 v}^{I}{ }^{2}-\Omega^{2}$ in equation [9], which can be written as follows:

$$
\left\|X_{i}^{I}(\Omega)\right\| \approx\left|\sum_{v=1}^{m} \frac{\Phi_{i v}^{I} \cdot \sum_{j=1}^{k} \Phi_{j v}^{I} \cdot F_{j}^{I}}{\omega_{0 v}^{I}{ }^{2}-\Omega^{2}}\right|
$$

Maximizing Equation [10] (respectively minimizing) comes down to maximizing (respectively minimizing) the numerator and minimizing (respectively maximizing) the denominator. Since the numerator is a sum of positive and negative terms, it is not possible to determine the parameter value combinations analytically. The choice of bounds for the intermediary zone is thus determined using interval arithmetic, since there is no problem due to multiple occurrences. 
For the resonance zone, the response of mode $v$ becomes dominant. The extreme levels are given for $\Omega=\omega_{0 \mu}$ with $\omega_{0 \mu}$ included between the lower and upper bounds of the pulsation $\omega_{0 \mu}{ }^{\alpha}$. In addition, $\omega_{0 v}^{I^{2}}-\Omega^{2}$ becomes negligible according to $2 . \xi_{v}^{I} \cdot \omega_{0 v}^{I} . \Omega$ in equation [9]. Thus, equation [9] can be transformed as follows:

$$
\left\|X_{i}^{I}(\Omega)\right\|=\left|\frac{\Phi_{i \mu}^{I} \cdot \sum_{j=1}^{k} \Phi_{j \mu}^{I} \cdot F_{j}^{I}}{2 \cdot \xi_{\mu}^{I} \cdot \Omega^{2}}+\sum_{\substack{v=1 \\ v \neq \mu}}^{m} \frac{\Phi_{i v}^{I} \cdot \sum_{j=1}^{k} \Phi_{j v}^{I} \cdot F_{j}^{I}}{\omega_{0 v}^{I}-\Omega^{2}}\right|
$$

Equation [11] allows the bounds of the parameters that can be taken into account to be determined. The interval solutions are again obtained using interval arithmetic.

For the antiresonance zone, the minimum level of FRFs boundaries is equal to the minimum value obtained when calculating the different FRFs.

Thus, as shown above, by coupling the functional study and the reanalysis technique, it is possible to extend the IFEM to the case of dynamic frequency response.

\section{Comparison of numerical and experimental results}

The objective of our methodology is to numerically predict the variation of a structure's dynamic behavior using knowledge of the nominal model and estimations of the different variability parameters. This numerical prediction was validated experimentally through experimental modal analysis. The test structure (Figure 5) consists of two honed steel plates, held together with eight Chc M4 bolts and then glued to avoid assembly defects. The finite element model of the nominal structure contains 400 shell elements and 2706 degrees of freedom. Imperfections were considered for 6 parameters: Young modulus E $( \pm 3 \%)$, plate thicknesses $\mathrm{e}_{1}$ and $\mathrm{e}_{2}$ $( \pm 20 \%)$ and sizes $\mathrm{D}_{1}, \mathrm{D}_{2}$ and $\mathrm{D}_{3}( \pm 1 \%)$.
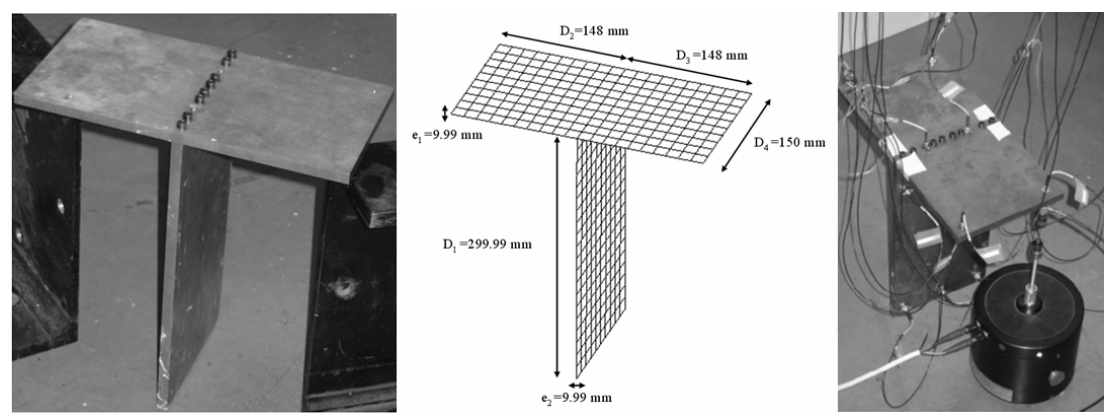

Figure 5. Nominal two-plate steel structure and the associated finite element model 
The numerical interval solutions were calculated using the Interval Finite Element Method, described in Section 3 for eigensolutions and in section 4 for FRFs. The experimental interval solutions were obtained by aggregating the results from several experimental modal analyses performed for the nominal values and the bounds of the interval variation for the two plate thicknesses. A total of nine $(3 \times 3)$ test structures were measured. The interval databases were built following a rigorous protocol designed to reduce the influence of experimental and modeling imperfections. (More details about the experimental tests are presented in Massa, 2005).

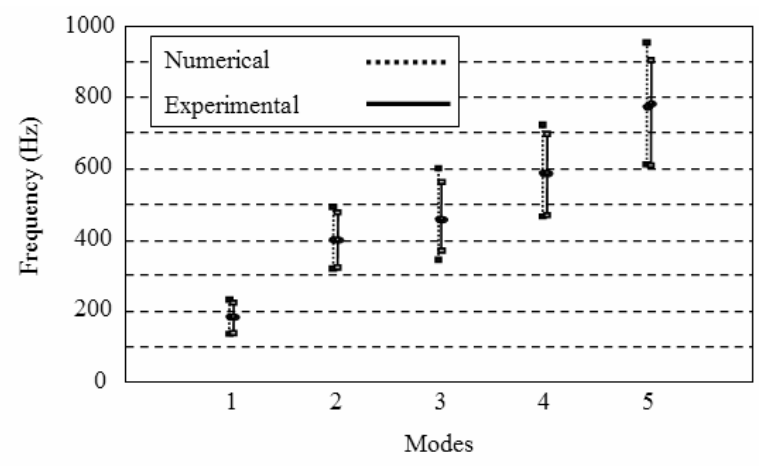

Figure 6. Spectrum of interval eigenfrequencies

Figure 6 presents the interval variations associated to the first five eigenfrequencies for both the numerical and experimental procedures. The numerical results come reasonably close to the experimental data. The maximal error made in evaluating the frequency bounds is less than $7 \%$. The worst result was obtained for the fifth eigenfrequency, because both the model and the measurements for this mode shape contain some errors.

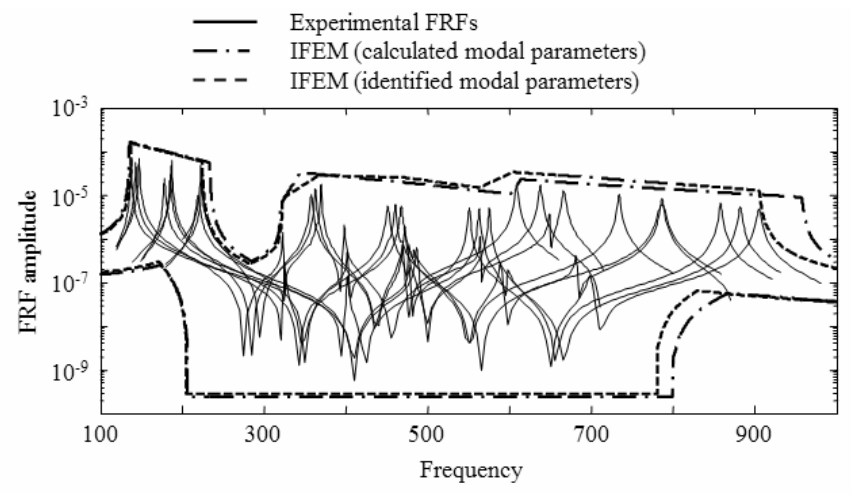

Figure 7. Experimental and numerical interval FRFS 
Figure 7 shows the nine FRFs obtained by experimental modal analysis compared to the numerical results obtained by IFEM. The results obtained for the lower and upper bounds of the FRFs are quite interesting: the amplitude of the dynamic response is correctly evaluated.

\section{Conclusion}

This paper proposed an Interval Finite Element Method for analyzing Frequency Response Functions. After studying the static and modal interval cases, this new investigation required a functional study, a sensitivity study and the use of a reanalysis technique using projection to approximate perturbed eigensolutions.

The numerical interval results were compared with an experimental interval reference. The experimental interval database was obtained by aggregating the results (frequencies and FRFs) from different configurations of the test structure. Using a good quality nominal model and a reasonable estimate of the variability of the significant parameters, we were able to demonstrate a good correspondence between the numerical and experimental data.

The main idea is to provide designers with calculation tools (e.g., static interval analysis, modal interval analysis, dynamic interval analysis) for different structural analysis studies. The proposed methodology determines the boundaries of a structure's behavioural variation using knowledge of a nominal model and estimations of the different variability parameters. The interval results will then introduce a notion of robustness in design optimization strategy.

\section{References}

Alefeld G., Herzberber J., Introductions to Interval Computations, Academic Press, New York, 1983.

Balmès E., Barthe D., Ravary F., «Propagation de méconnaissances en analyse modale », $\sigma^{e}$ colloque national en calcul des structures, Giens, France, 2003, p. 407-414.

Balmès E., Ravary F., Langlais D., "Uncertainty propagation in modal analysis", Proceedings of $24^{\text {th }}$ International Modal Analysis Conference, 2005, Orlando.

Capiez-Lernout E., Pellissetti M., Pradlwarter H., Schueller G.I., Soize C., "Data and model uncertainties in complex aerospace engineering systems", Journal of Sound and Vibration, vol. 295, 2006, p. 923-938.

Cochelin B., Damil N., Potier-Ferry M., "Asymptotic numerical methods and Padé approximants for non linear elastic structures”, Revue Européenne des Eléments finis, vol. $3, n^{\circ} 2,1994$, p. 281-297. 
De Gersem H., Moens D., Desmet W., Vandepitte D., "Interval and fuzzy dynamic analysis of finite element models with superelements", Computers and Structures, vol. 85, 2007, p. 304-319.

Lee I.-W., Jung G.-H., “An efficient algebraic method for the computation of natural frequency and mode shape sensitivities - Part I. Distinct natural frequencies", Computers and Structures, vol. 62, n 3, 1997, p. 429-435.

Massa F., Lallemand B., Tison T., «Comportement statique et modal des structures en présence de variabilités paramétriques $", \sigma^{e}$ colloque national en calcul des structures, Giens, France, 2003, p. 415-422.

Massa F., Gestion des imperfections en phase de conception des structures mécaniques, Thèse de doctorat, Université de Valenciennes, 2005.

Massa F., Tison T., Lallemand B., "A fuzzy procedure for the static design of imprecise structures", Computer Methods in Applied Mechanics and Engineering, vol. 195, 2006, p. $925-941$.

Massa F., Ruffin K., Tison T., Lallemand B., “A complete method for efficient fuzzy modal analysis", Journal of Sound and Vibration, vol. 309, n 1-2, 2008, p. 63-85.

McWilliam S., "Anti-optimisation of uncertain structures using interval analysis”, Computers and Structures, vol. 79, 2001, p. 421-430.

Moore R.E., Interval Analysis, Prentice Hall, Englewood Cliffs, New York, 1966.

Mourier-Ruffin K., Massa F., Tison T., Lallemand B., « Réponse harmonique de structures comportant des paramètres imprécis ", $8^{e}$ colloque national en calcul des structures, Giens, France, 2007.

Sudret B., Der Kiureghian A., Stochastic finite element methods and reliability: a state-of-theart report», Technical Report UCB/SEMM-2000/08, University of California, 2000. 